\title{
Dynamical Masses for the Large Magellanic Cloud Massive Binary System [L72] LH 54-425 ${ }^{1}$
}

\author{
S. J. Williams, D. R. Gies, T. J. Henry \\ Center for High Angular Resolution Astronomy and \\ Department of Physics and Astronomy, \\ Georgia State University, P. O. Box 4106, Atlanta, GA 30302-4106; \\ swilliams@chara.gsu.edu,gies@chara.gsu.edu,thenry@chara.gsu.edu \\ J. A. Orosz \\ Department of Astronomy, San Diego State University, 5500 Campanile Drive, San Diego, \\ CA 92182-1221; orosz@sciences.sdsu.edu \\ M. V. McSwain \\ Department of Physics, Lehigh University, 16 Memorial Drive East, Bethlehem, PA 18015; \\ mcswain@lehigh.edu \\ T. C. Hillwig \\ Department of Physics and Astronomy, Valparaiso University, Valparaiso, IN, 46383; \\ todd.hillwig@valpo.edu \\ L. R. Penny \\ Department of Physics and Astronomy, College of Charleston, 101 Science Center, 58 \\ Coming Street, Charleston, SC 29424; pennyl@cofc.edu \\ G. Sonneborn \\ NASA Goddard Space Flight Center, Code 681, Greenbelt, MD 20771; \\ george.sonneborn@nasa.gov \\ R. Iping \\ Department of Physics, Catholic University of America, Washington, DC 20064; and \\ NASA Goddard Space Flight Center, Code 665, Greenbelt, MD 20771; \\ Rosina.C.Iping@nasa.gov \\ K. A. van der Hucht
}


SRON Netherlands Institute for Space Research, Sorbonnelaan 2, 3584CA Utrecht, The

Netherlands; Astronomical Institute Anton Pannekoek, University of Amsterdam, Kruislaan 403, 1097 SJ Amsterdam, The Netherlands; k.a.van.der.hucht@sron.nl

\title{
L. Kaper
}

Astronomical Institute, University of Amsterdam, Kruislaan 403, 1098 SJ Amsterdam, The Netherlands; lexk@science.uva.nl

\begin{abstract}
We present results from an optical spectroscopic investigation of the massive binary system [L72] LH 54-425 in the LH 54 OB association in the Large Magellanic Cloud. We revise the ephemeris of [L72] LH 54-425 and find an orbital period of $2.24741 \pm 0.00004$ days. We find spectral types of O3 $\mathrm{V}$ for the primary and $\mathrm{O} 5 \mathrm{~V}$ for the secondary. We made a combined solution of the radial velocities and previously published $V$-band photometry to determine the inclination for two system configurations, $i=52_{-3}^{+2}$ degrees for the configuration of the secondary star being more tidally distorted and $i=55 \pm 1$ degrees for the primary as the more tidally distorted star. We argue that the latter case is more probable, and this solution yields masses and radii of $M_{1}=47 \pm 2 M_{\odot}$ and $R_{1}=11.4 \pm 0.1 R_{\odot}$ for the primary, and $M_{2}=28 \pm 1 M_{\odot}$ and $R_{2}=8.1 \pm 0.1 R_{\odot}$ for the secondary. Our analysis places LH 54-425 amongst the most massive stars known. Based on the position of the two stars plotted on a theoretical HR diagram, we find the age of the system to be $\sim 1.5 \mathrm{Myr}$.
\end{abstract}

Subject headings: binaries: spectroscopic - stars: early-type - stars: fundamental parameters - stars: individual ([L72] LH 54-425)

\section{Introduction}

One of the most elusive of important parameters in stellar astrophysics is the mass of stars. Stars in binary or multiple systems, and specifically in double-lined spectroscopic

\footnotetext{
${ }^{1}$ Based on observations with the CTIO SMARTS Consortium 1.5-meter telescope and the 2.5-meter DuPont telescope at Las Campanas.
} 
binaries, offer us a chance to explore their masses with one important caveat: we must know the orbital inclination of the system. The inclination is the key that allows the resolution of the spectroscopic results into individual masses for the members of the system. Individual stellar masses are obtained via the serendipitous orientation of the system for eclipses or, at the very least, ellipsoidal light variations. Particularly rare are O-type main-sequence systems, owing partly to their short lifespans and partly to the steepness of the initial mass function. Reported here is the first of several systems to be investigated with the goal of determining the masses, radii, and distances to a number of O-star binaries. Mass data of this kind are needed to help resolve the mass discrepancy problem between the evolutionary and spectroscopic mass estimates for massive stars (Repolust et al. 2004; Massey et al. 2005).

The earliest type star in the Large Magellanic Cloud LH 54 OB association is [L72] LH 54-425 (Hill et al. 1994), also known as L54S-4 (Oey 1996). Its spectral type was classified by Oey \& Smedley (1998) as O4 III(f*). Its binary nature was unknown until Ostrov (2002) noted the ellipsoidal variations in the $V$-band photometry and obtained six spectroscopic observations of the system. The light and radial velocity curves from that study were used to estimate the masses of the two stars at $100 M_{\odot}$ and $50 M_{\odot}$. The lack of phase coverage for the radial velocity data did not allow for a more rigorous analysis of physical parameters.

We present here an analysis of the radial velocities from optical spectra obtained between 2003 and 2006 (§2). In $\S 3$ we discuss how we extracted radial velocities from the observations and solved for the orbital parameters. We describe tomographic reconstructions of the individual component spectra in $\S 4$. The next section $(\S 5)$ covers our combined light curve and radial velocity solution, as well as our determination of the inclination of the system for two system configurations. We conclude with a discussion of the system's evolutionary status, fundamental parameters, and a comparison of the two model fits to the system in $\S 6$.

\section{Observations}

The first sets of spectroscopic data were obtained from 2003 through 2006 with the Cerro Tololo Inter-American Observatory (CTIO) 1.5-m telescope and RC spectrograph operated by the SMARTS Consortium. We used grating \#47 (831 lines per mm, $8000 \AA$ blaze wavelength in the first order Littrow configuration) in second order (SMARTS configuration 47/IIb) together with a BG39 or $\mathrm{CuSO}_{4}$ order blocking filter. The detector was a Loral $1200 \times 800 \mathrm{CCD}$. This arrangement produced spectra covering the range $4058-4732 \AA$ with a resolving power of $R=\lambda / \Delta \lambda \approx 3550$. Exposure times were 1800 seconds, in order to reach a moderate signal-to-noise ratio $(\mathrm{S} / \mathrm{N} \approx 70)$. Each observation was bracketed with $\mathrm{HeAr}$ comparison spectra for wavelength calibration, and numerous bias and flat field spectra were 
obtained each night. We obtained a total of 48 spectra of the target with this configuration.

In addition to the CTIO data, four observations were obtained with the echelle spectrograph on the 2.5-m DuPont telescope at Las Campanas Observatory binned $2 \times 2$ with a resolving power of $\approx 27000$ (from measurements of arc spectra). Exposures were combined where there were two, and the spectral flux was normalized. Orders were merged and cosmic rays removed. For continuity of analysis, these data were clipped and re-sampled to match the wavelength and lower resolution CTIO data.

The resulting spectra were extracted and wavelength calibrated using standard routines in IRAF2. All spectra were then rectified to a unit continuum via fitting line-free regions and transformed to a common heliocentric wavelength grid in $\log \lambda$ increments.

\section{Radial Velocities and Orbital Elements}

Radial velocities were measured using a template-fitting scheme (Gies et al. 2002) for the five lines $\mathrm{H} \delta \lambda 4101$, He II $\lambda 4200, \mathrm{H} \gamma \lambda 4340$, He II $\lambda 4542$, and He II $\lambda 4686$. These were the only prominent lines in the spectrum of each star, and no single line showed any systematic shift from the others. The spectra show no evidence for wind effects in the lines used to derive the orbital solution. No clear emission features and no asymmetries of absorption features were seen in the hydrogen lines or the He II $\lambda 4686$ line. The basic procedure followed to obtain radial velocities is outlined in Boyajian et al. (2007).

The spectrum from HJD 2,452,718.5370 shows well separated spectral features from each star in the He II $\lambda \lambda 4542,4686$ lines. These lines were used to derive matching template spectra for the primary and secondary components. Template spectra were obtained from the grid of O-type stellar models from Lanz \& Hubeny (2003), which are based on the line blanketed, non-LTE, plane-parallel, hydrostatic atmosphere code TLUSTY and the radiative transfer code SYNSPEC (Hubeny 1988; Hubeny \& Lanz 1995; Hubeny et al. 1998). In order to find matching templates, approximations were initially used for the temperatures, gravities, projected rotational velocities, and flux contributions from each star. These initial parameters for model templates were then checked after velocity analysis by studying the properties of the tomographically reconstructed spectra of the components $(\S 4)$.

Further inputs for the template fitting scheme are preliminary estimates of the velocities

\footnotetext{
${ }^{2}$ IRAF is distributed by the National Optical Astronomy Observatory, which is operated by the Association of Universities for Research in Astronomy, Inc., under cooperative agreement with the National Science Foundation.
} 
for the primary and secondary. The He II $\lambda 4542$ line for each star was fit using Gaussian functions with the splot routine and deblend option in IRAF. Radial velocity shifts were then calculated for all spectra with well separated lines, and these were used to obtain initial orbital parameters. The preliminary velocity from the initial orbital solution gave a starting point to perform a non-linear, least squares fit of the composite profiles with the template spectra and calculate the shifts for each star. The five values for radial velocity from each set of five lines in an individual spectrum were then averaged to obtain a mean value, and the standard deviation of the mean value was calculated. Each of these values are listed in Table 1 for the primary and secondary stars, as well as the orbital phase for each observation and the observed minus calculated values for each point. Zero phase is defined by the time of inferior conjunction of the primary star, $T_{\mathrm{IC}, 1}$.

We made an orbital fit of the radial velocity data using the non-linear, least-squares fitting program of Morbey \& Brosterhus (1974). Equal weights were assigned to each data point, because all the spectra were comparable in $\mathrm{S} / \mathrm{N}$ ratio, spectral coverage, resolving power, and quality of radial velocities derived. It should be noted that for massive binaries, discrepancies in systemic velocities between components may be attributed to differences in their expanding atmospheres and/or differences in the shapes of template spectral lines, so we fit systemic velocities for each component. Fits with non-zero eccentricities were explored, none of which gave as good a fit as a circular orbital solution. Fits were also made for subsets of measurements near conjunction (phases near 0.0 and 0.5) and quadrature (phases near 0.25 and 0.75 ). These tests revealed that the important spectroscopic elements, such as the velocity semi-amplitudes, did not vary by more than $3 \sigma$ from the best fits for any subset. Our results for the orbit using spectroscopic data only are listed in column 3 of Table 2. The radial velocity measurements and final orbital velocity curves (§5) are shown in Figure 1,

\section{Tomographic Reconstruction}

We used a Doppler tomography algorithm (Bagnuolo et al. 1994) to separate the primary and secondary spectra of [L72] LH 54-425. This is an iterative method that uses the 48 observed composite spectra from CTIO, their velocity shifts, and an assumed monochromatic flux ratio $\left(F_{2} / F_{1}\right)$ to derive the individual component spectra. We explored a range of flux ratios to arrive at a value that best matched the line depths in the reconstructions with those in model spectra. Figure 2 shows the reconstructed spectra for the primary and secondary, as well as synthetic spectra just below each. Few lines are present in our spectra. However, the relative strength of the He I $\lambda 4471$ in the secondary is the major difference between the two spectra and indicates the cooler temperature of the secondary. 
These reconstructed spectra were fit with TLUSTY/SYNSPEC model synthesis spectra (see $\S 3$ ). These matches allow for estimates of stellar parameters listed in Table 3 , The width of the He II $\lambda 4542$ line was used to estimate the projected rotational velocity $V \sin i$ for each star by comparing the reconstructed and model profiles for a grid of test $V \sin i$ values. Matches were made to the $\mathrm{H} \gamma \lambda 4340$ and He I $\lambda 4471$ lines to obtain the temperature and surface gravity estimates. Finally, we used the ratios of the reconstructed to synthetic spectral line depths to estimate that the monochromatic flux ratio $\left(F_{2} / F_{1}\right)$ in our blue spectra is $0.45 \pm 0.10$.

A visual inspection of our reconstructed spectra match best the $\mathrm{O} 3 \mathrm{~V}$ example in Figure 6 of Walborn et al. (2002) for the primary and the O5 V example in Figure 7 of Walborn et al. (2002), and these are the spectral types we list in Table 3. Unfortunately, our wavelength coverage does not include the N IV $\lambda 4058$ feature suggested in Walborn et al. (2002) for spectral classification of the hottest O-stars. The N III $\lambda 4634-42$ feature is another good diagnostic for classification of early O-type stars, but is too weak in our spectra for measurement. We can use the equivalent width ratio of He I $\lambda 4471 /$ He II $\lambda 4542$ calibrated in Mathys (1988) for each of our reconstructed spectra to obtain a spectral class. This measurement results in a spectral class for the primary of O3 and of O5.5 for the secondary. We used our best matched effective temperatures from the TLUSTY/SYNSPEC models combined with the $T_{\text {eff }}$ versus spectral type calibration of Martins et al. (2005) to estimate spectral types of $\mathrm{O} 3 \mathrm{~V}$ for the primary and $\mathrm{O} 5 \mathrm{~V}$ for the secondary. These spectral types and effective temperatures are also consistent with those shown in Figure 1 of Mokiem et al. (2007) for LMC O-type dwarfs.

\section{Combined Radial Velocity and Light Curve Solution}

Light curve data were taken from the $V$-band observations listed in Table 1 of Ostrov (2002). New orbital parameters were then found using the Eclipsing Light Curve (ELC) code (Orosz \& Hauschildt 2000), which fits the radial velocity and light curves simultaneously. The resultant light curve is shown in Figure 3, and the radial velocity curves are shown in Figure 1, along with the radial velocity measurements from Ostrov (2002), which were not used for any fit in this paper. ELC's genetic optimizer was used to explore the parameter space and was given wide ranges for each value of period, epoch of inferior conjunction of the primary $T_{\mathrm{IC}, 1}$, inclination, mass ratio, primary velocity semi-amplitude, and Roche-lobe filling factor for each star. The Roche-lobe filling factor is defined as the ratio of the radius of the star toward the inner Lagrangian Point $\left(L_{1}\right)$ to the distance to $L_{1}$ from the center of the star, $f \equiv x_{\text {point }} / x_{L 1}$ (Orosz \& Hauschildt 2000); our fits show that our stars do not fill 
their Roche lobe (see Table 4). A by-product of the exploration of parameter space by ELC was a determination of systemic velocities for each component.

The temperatures of each star were set and not fit because these were well constrained by analysis of the reconstructed spectra discussed in $\S 4$. As with the fits of only radial velocities discussed in $\S 3$, circular orbital solutions proved best.

To estimate the 1,2 , and $3 \sigma$ uncertainties associated with the fitted parameters, we followed the method discussed in Orosz et al. (2002). The values of the seven fitted parameters (plus the two systemic velocities) listed above were varied in the calculation of $\sim 3.6 \times 10^{6}$ light and radial velocity curves. This procedure resulted in thorough sampling near the $\chi_{\min }^{2}$ point, making the multi-dimensional surface well populated. This allowed for projection of the $\chi^{2}$ surface as a function of each parameter or fitted value of interest. The lowest $\chi^{2}$ is then found for each parameter of interest, and the 1,2, and $3 \sigma$ uncertainties may be estimated by the regions where $\chi^{2} \leq \chi_{\min }^{2}+1,+4$, and +9 , respectively. The uncertainties listed in the tables are $1 \sigma$ uncertainties.

Special attention was paid to the inclination of the system. The well-sampled light curve from Ostrov (2002) allows for an exploration of the important parameters that cause the ellipsoidal variations in this system: inclination and Roche-lobe filling factors for each star. Ellipsoidal light variations occur in systems where one or both of the components are tidally distorted due to the proximity of its companion. At different orbital phases, the observer sees varying amounts of light from the system, based upon the interplay between the amount of tidal distortion, the observed cross-section of the two stars, and the inclination of the system. As was expected, we found that for lower inclinations, i.e., viewing the system more face-on, the sizes and tidal distortions of the stars needed to become larger in order to match the modulation of the light curve. Thus, restricting the stars to radii within their Roche lobes ultimately limits the lowest acceptable inclination, while the lack of observed eclipses establishes the maximum inclination.

Again, using the genetic algorithm in ELC, we explored the range of inclination values suggested in Ostrov (2002) and allowed the fill factors for each star to vary over a wide range while keeping the other parameters fixed. The results of this analysis are plotted as contours of 1,2, and $3 \sigma$ confidence intervals in Figures 4 and 5 . In the best overall fit, the estimated filling factors are larger for the secondary, so most of the ellipsoidal variation is due to the tidal distortion of the secondary (Fig. 44). It is interesting to note that the contours suggest a rather steep surface toward higher inclinations, indicating a hard upper limit for the inclination of $56^{\circ}$ at the $3 \sigma$ level. Lower inclinations are not as probable, and fill factors for the secondary get too large to match the light curve as the secondary star approaches a fill factor of 1.0, thus completely filling its Roche-lobe. These characteristics 
help us to constrain the inclination of the system to be $i=52_{-3}^{+2}$ degrees. The corresponding masses and other parameters are listed in Table 4. Included are values for $V_{\text {sync }} \sin i$, the projected rotational velocity assuming synchronous rotation, $R_{\text {eff }}$, the effective radius for a sphere of the same volume, and the sizes of the stars along different directions.

The orbital parameters for the best fit are listed in Table 2. The spectroscopic solution and the combined solution from ELC match very well. It should be noted that the parameters in Table 4 with asymmetric uncertainties are a result of the asymmetry in the uncertainties for the inclination.

We also explored the possibility of the primary being the more tidally distorted star. To do this we ran the simulations again, keeping the same parameters from Table 2 and varying only the filling factors and inclination. Thus, the radial velocity curve does not change and the light curve is fit to roughly the same degree of accuracy as before, so the fits in Figures 1 and 3 need not be changed. The contour plot for this scenario, again with confidence intervals of 1,2 , and $3 \sigma$, is shown in Figure 5. The fit gives an inclination of $i=55 \pm 1$ degrees. This corresponds to slightly different values of masses and radii, listed alongside the overall best fit in Table 4. The small range in inclination leads to a small range in the masses and radii of the components of the system. This restricted fit has a reduced $\chi^{2}$ of 2.69 versus 2.54 for the case where the secondary is more tidally distorted.

A contact binary is another possible configuration for the system. We did not explore this possibility because in a contact system, the sum of the values of $V \sin i$ and the sum of the velocity semi-amplitudes would be comparable. For our fits of [L72] LH 54-425 (see Tables 2 and 3 ), the sums of these values differ by at least $300 \mathrm{~km} \mathrm{~s}^{-1}$.

\section{Discussion and Conclusions}

Which of our two models is most consistent with the available data? The answer to this question lies in comparing the consequences of each solution.

To begin the comparison, we used the temperatures and radii output from ELC to obtain an estimate of the monochromatic flux ratio $F_{2} / F_{1}$. The case where the secondary is more tidally distorted yields a model flux ratio of $0.70{ }_{-0.01}^{+0.05}$, while the primary as the more tidally distorted star gives a flux ratio of $0.46 \pm 0.02$, in agreement with the value of $0.45 \pm 0.05$ derived from the tomographic reconstructions.

Theory suggests (Ekström et al. 2008) that for massive stars, the main sequence radius scales as $R \sim M^{0.6}$. Applying this as another consistency check, we can substitute velocity 
semi-amplitudes to estimate the expected ratio of radii, $R_{2} / R_{1} \sim\left(K_{1} / K_{2}\right)^{0.6}$ which, from our values in Table 2 is $0.707 \pm 0.10$. The ratio of effective radii estimated from the output of ELC (Table 4) is $\sim 0.88_{-0.05}^{+0.03}$ for the fit with the secondary as the more tidally distorted and $\sim 0.71 \pm 0.010$ for the primary being more tidally distorted. Once again the fit with the primary as the more tidally distorted matches theory more closely.

Ignoring the tidal distortions, we can estimate the luminosity by applying the StefanBoltzmann equation $L=4 \pi R^{2} \sigma T_{\text {eff }}^{4}$, and combining this with our temperature measurements, place each component in a theoretical H-R diagram. This is presented in Figure 6, along with evolutionary tracks from Schaerer et al. (1993) for stars of varying mass with a metallicity of $Z=0.008$ which is appropriate for the LMC. The $1 \sigma$ uncertainty regions are consistent with an age of $\sim 1.5 \mathrm{Myr}$ for the model consisting of the primary as the more tidally distorted star, and both stars appear to be co-evolutionary. The tidally distorted secondary fit appears a bit older, roughly matching the $2 \mathrm{Myr}$ isochrone. This fit also indicates that the secondary is more evolved than the primary and is overluminous for its derived mass. Therefore, it seems the fit with the primary being more tidally distorted is more consistent with evolutionary tracks. These approximate ages are also consistent within uncertainties with the estimate from Oev \& Smedley (1998) of 2-3 Myr for the LH 54 OB association. According to Table 1 of Schaerer et al. (1993), even the oldest age of $\sim 2$ Myr puts [L72] LH 54-425 in the part of its life when it is still burning hydrogen, and it will continue to do so for another $\sim 1.5-2$ Myr.

For the next comparison, we used the luminosities calculated above to obtain a bolometric absolute magnitude, assuming a solar bolometric absolute magnitude of $4.74 \mathrm{mag}$ (Cox 2000). Next, we applied a bolometric correction for each star based on our derived spectral types from Martins et al. (2005) to obtain absolute $V$ magnitudes. We then combined these magnitudes to obtain an absolute $V$ magnitude for the system. Finally, we arrived at a distance modulus for the system by using the maximum magnitude of the system from the photometric data and $E(B-V)=0.10 \mathrm{mag}$ (Oey \& Smedley 1998). The resulting distance modulus from the fit with the more tidally distorted secondary is 18.65 mag while the distance modulus for the tidally distorted primary fit is $18.55 \mathrm{mag}$. Both of these numbers are consistent with the HST Key Project distance modulus to the LMC of $18.50 \pm 0.10 \mathrm{mag}$ (Freedman et al. 2001), with the tidally distorted primary fit being slightly better. Note that each of the contour plots in Figures 4 and 5 seem to have a best fit valley in the $\chi^{2}$ as a function of inclination and filling factor. As one travels along this valley to higher inclinations, the dimensions of the system get smaller, including the sizes of the two stars. This makes the stars intrinsically fainter, and they must lie at a closer distance to match the observed $V$ photometry. In contrast, the lower inclinations mean the stars become bigger and lie at greater distances. The overall agreement in distance modulus between the model 
results and those for the LMC indicate that the final fill out estimates are reliable.

The two models are most different in the $V_{\text {sync }} \sin i$ values output from ELC. The observed $V \sin i$ values from the reconstructed spectra are $197 \pm 5 \mathrm{~km} \mathrm{~s}^{-1}$ for the primary and $182 \pm 8 \mathrm{~km} \mathrm{~s}^{-1}$ for the secondary. The ELC fit for the more distorted secondary gives values of $197 \pm 5 \mathrm{~km} \mathrm{~s}^{-1}$ and $173 \pm 7 \mathrm{~km} \mathrm{~s}^{-1}$ for the primary and secondary, respectively, while the ELC fit for the more distorted primary gives $209 \pm 5 \mathrm{~km} \mathrm{~s}^{-1}$ and $148 \pm 6 \mathrm{~km} \mathrm{~s}^{-1}$. Both cases agree within uncertainties for the primary's line broadening, but the predicted $V \sin i$ for the secondary is too small for the case where the primary is the more tidally distorted star. However, due to the youth of the system, our assumption of synchronous rotation may not be appropriate. There may not have yet been enough time for tidal forces to synchronize the spin with the orbit. More ELC fits were performed assuming a faster rotation for the secondary and these show that the same physical parameters are obtained for a non-synchronous rotation scenario.

Given the above tests, we think the fit with the primary as the more tidally distorted star is the more likely configuration. For this case, the masses and radii are $M_{1}=47 \pm 2 M_{\odot}$ and $R_{1}=11.4 \pm 0.1 R_{\odot}$ for the primary and $M_{2}=28 \pm 1 M_{\odot}$ and $R_{2}=8.1 \pm 0.1 R_{\odot}$ for secondary. These masses are significantly less than the $100 M_{\odot}$ and $50 M_{\odot}$ values obtained by Ostrov (2002). Part of the reason lies in the data set we obtained for radial velocities of the system. As is shown in Figure 1, two secondary velocity values derived by Ostrov (2002) near phase 0.25 are the ones that drove the estimate for the velocity semi-amplitude up, and consequently the mass ratio down. This, in addition to the lower inclination adopted by Ostrov (2002), leads to the differences in masses between our two analyses. Our temperatures are also slightly lower than those given in Ostrov (2002, see his Table 3), due to his use of a different, older temperature versus spectral-type calibration (Aller et al. 1982). We can compare our primary star with the primary in R136-38 (Massey et al. 2002), which is also an O3 V. Massey et al. (2002) derive a mass for their O3 V star of $56.9 \pm 0.6 M_{\odot}$, in closer agreement with our value of $47 M_{\odot}$ than that of $100 M_{\odot}$ found by Ostrov (2002). Our derived radius for the primary in LH 54-425 is slightly larger than that for the primary in R136-38 of $9.3 R_{\odot}$ (Massey et al. 2002). This may be due to the extreme youth of R136-38, which appears to be near the zero-age main sequence (see Fig. 6 in Massey et al. 2002), while the position of [L72] LH 54-425 in the H-R diagram (our Fig. 6) indicates the primary is slightly evolved. These numbers are consistent with the fact that the LH $54 \mathrm{OB}$ association, at $\sim 2-3$ Myr (Oev \& Smedlev 1998), is slightly older than the R136 cluster with an age of 1-2 Myr (Massey \& Hunter 1998).

We gratefully acknowledge the referee for comments and suggestions that improved the quality and organization of the paper. We also gratefully acknowledge the 2.5-meter DuPont 
data and thoughtful comments and suggestions generously offered by Alceste Bonanos. This material is based upon work supported by the National Science Foundation under Grant No. AST-0506573 and AST-0606861. We gratefully acknowledge support from the GSU College of Arts and Sciences and from the Research Program Enhancement fund of the Board of Regents of the University System of Georgia, administered through the GSU Office

of the Vice President for Research. We also wish to thank Fred Walter and the SMARTS Consortium for a generous allocation of time to study this system with the CTIO 1.5-m telescope.

\section{REFERENCES}

Aller, L. H., et al. 1982, Landolt-Bornstein: Numerical Data and Functional Relationships in Science and Technology (Springer-Verlag: New York)

Bagnuolo, W. G., Jr., Gies, D. R., Hahula, M. E., Wiemker, R., \& Wiggs, M. S. 1994, ApJ, 423,446

Boyajian, T. S., et al. 2007, ApJ, 664, 1121

Cox, A. N. 2000, Allen's Astrophysical Quantities, 4th ed. (New York: AIP Press)

Ekström, S., Meynet, G., Maeder, A., \& Barblan, F. 2008, A\&A, 478, 467

Freedman, W. L., et al. 2001, ApJ, 553, 47

Gies, D. R., Penny, L. R., Mayer, P., Drechsel, H., \& Lorenz, R. 2002, ApJ, 574, 957

Hill, R. J., Madore, B. F., \& Freedman, W. L. 1994, ApJS, 91, 583

Hubeny, I. 1988, Computer Physics Communications, 52, 103

Hubeny, I., Heap, S. R., \& Lanz, T. 1998, in Boulder-Munich II: Properties of Hot Luminous Stars, (ASP Conf. Ser. 131), ed. I. Howarth (San Francisco: ASP), 108

Hubeny, I., \& Lanz, T. 1995, ApJ, 439, 875

Lanz, T., \& Hubeny, I. 2003, ApJS, 146, 417

Lejeune, T., \& Schaerer, D. 2001, A\&A, 366, 538

Martins, F., Schaerer, D., \& Hillier, D. J. 2005, A\&A, 436, 1049

Massey, P., \& Hunter, D. A. 1998, ApJ, 493, 180 
Massey, P., Penny, L. R., \& Vukovich, J. 2002, ApJ, 565, 982

Massey, P., Puls, J., Pauldrach, A. W. A., Bresolin, F., Kudritzki, R. P., \& Simon, T. 2005, ApJ, 627, 477

Mathys, G. 1988, A\&AS, 76, 427

Mokiem, M. R., et al. 2007, A\&A, 465, 1003

Morbey, C. L., \& Brosterhus, E. B. 1974, PASP, 86, 455

Oey, M. S., \& Smedley, S. A. 1998, AJ, 116, 1263

Oey, M. S. 1996, ApJS, 104, 71

Orosz, J. A., \& Hauschildt, P. H. 2000, A\&A, 364, 265

Orosz, J. A., et al. 2002, ApJ, 568, 845

Ostrov, P. G. 2002, MNRAS, 336, 309

Repolust, T., Puls, J., \& Herrero, A. 2004, A\&A, 415, 349

Schaerer, D., Meynet, G., Maeder, A., \& Schaller, G. 1993, A\&AS, 98, 523

Walborn, N. R., et al. 2002, AJ, 123, 2754 
Table 1. LH 54-425 Radial Velocity Measurements

\begin{tabular}{|c|c|c|c|c|c|c|c|}
\hline $\begin{array}{c}\text { Date } \\
(\text { HJD-2,400,000) }\end{array}$ & $\begin{array}{l}\text { Orbital } \\
\text { Phase }\end{array}$ & $\begin{array}{c}V_{1} \\
\left(\mathrm{~km} \mathrm{~s}^{-1}\right)\end{array}$ & $\begin{array}{c}\sigma_{1} \\
\left(\mathrm{~km} \mathrm{~s}^{-1}\right)\end{array}$ & $\begin{array}{l}(O-C)_{1} \\
\left(\mathrm{~km} \mathrm{~s}^{-1}\right)\end{array}$ & $\begin{array}{c}V_{2} \\
\left(\mathrm{~km} \mathrm{~s}^{-1}\right)\end{array}$ & $\left(\begin{array}{c}\sigma_{2} \\
\left(\mathrm{~km} \mathrm{~s}^{-1}\right)\end{array}\right.$ & $\begin{array}{l}(O-C)_{2} \\
\left(\mathrm{~km} \mathrm{~s}^{-1}\right)\end{array}$ \\
\hline 52714.5496 & 0.080 & 393.0 & 6.7 & -4.1 & 126.4 & 20.7 & -16.2 \\
\hline 52714.5756 & 0.092 & 398.5 & 12.9 & -11.1 & 72.8 & 49.6 & -47.5 \\
\hline 52714.6009 & 0.103 & 414.3 & 4.9 & -6.7 & 90.9 & 42.1 & -9.2 \\
\hline 52714.6312 & 0.116 & 430.9 & 11.7 & -3.1 & 91.2 & 14.6 & 14.0 \\
\hline 52714.6586 & 0.129 & 431.4 & 15.6 & -13.7 & 39.1 & 38.6 & -18.4 \\
\hline 52715.5251 & 0.514 & 269.6 & 9.7 & -11.8 & 405.9 & 16.7 & 57.1 \\
\hline 52715.5487 & 0.525 & 263.0 & 9.1 & -4.8 & 404.0 & 23.1 & 31.4 \\
\hline 52715.5725 & 0.535 & 231.3 & 5.7 & -23.8 & 423.6 & 11.5 & 28.5 \\
\hline 52715.5971 & 0.546 & 242.3 & 11.1 & 0.9 & 384.8 & 9.1 & -34.4 \\
\hline 52715.6216 & 0.557 & 213.4 & 5.0 & -14.5 & 418.4 & 14.3 & -24.4 \\
\hline 52716.5492 & 0.970 & 245.8 & 19.2 & -16.0 & 389.8 & 20.5 & 4.4 \\
\hline 52716.5722 & 0.980 & 234.2 & 17.4 & -40.4 & 398.1 & 17.8 & 35.6 \\
\hline 52716.5952 & 0.990 & 252.9 & 24.1 & -34.6 & 354.5 & 27.1 & 15.3 \\
\hline 52716.6182 & 0.000 & 343.9 & 31.6 & 43.4 & 231.1 & 35.6 & -84.7 \\
\hline 52717.5139 & 0.399 & 443.8 & 14.0 & 23.5 & 149.6 & 39.9 & 43.9 \\
\hline 52717.5370 & 0.409 & 419.3 & 16.6 & 10.1 & 147.8 & 39.3 & 22.5 \\
\hline 52717.5642 & 0.421 & 404.9 & 10.2 & 8.8 & 171.2 & 21.5 & 22.8 \\
\hline 52717.5878 & 0.432 & 387.5 & 15.0 & 3.5 & 183.0 & 23.3 & 13.3 \\
\hline 52717.6122 & 0.443 & 378.2 & 16.3 & 6.8 & 198.6 & 17.0 & 6.8 \\
\hline 52718.5139 & 0.844 & 139.1 & 6.4 & 5.6 & 621.2 & 55.7 & 7.8 \\
\hline 52718.5370 & 0.854 & 137.6 & 20.6 & -3.3 & 595.3 & 28.4 & -5.0 \\
\hline 52718.5600 & 0.864 & 142.0 & 9.3 & -7.0 & 563.3 & 26.1 & -22.8 \\
\hline 52718.5831 & 0.875 & 151.1 & 10.2 & -6.6 & 575.4 & 26.5 & 4.6 \\
\hline 52718.6060 & 0.885 & 157.9 & 7.4 & -9.2 & 536.5 & 18.1 & -17.8 \\
\hline 53017.5789 & 0.915 & 152.5 & 12.4 & -44.9 & 469.5 & 23.3 & -31.0 \\
\hline 53017.7698 & 0.000 & 323.6 & 25.6 & 23.8 & 259.5 & 36.7 & -57.5 \\
\hline 53018.5366 & 0.341 & 499.9 & 22.4 & 30.8 & 67.6 & 46.7 & 48.4 \\
\hline 53019.5645 & 0.798 & 102.2 & 11.9 & -6.7 & 654.1 & 12.7 & -1.9 \\
\hline 53019.7591 & 0.785 & 160.2 & 21.6 & -6.8 & 555.3 & 18.5 & 0.8 \\
\hline 53020.5382 & 0.232 & 515.8 & 11.4 & 17.4 & -10.0 & 42.2 & 24.9 \\
\hline 53021.5305 & 0.673 & 149.7 & 15.4 & 28.0 & 662.8 & 8.9 & 32.1 \\
\hline 53021.7435 & 0.768 & 104.3 & 6.8 & 3.2 & 670.6 & 3.7 & 1.5 \\
\hline 53022.5223 & 0.114 & 437.1 & 23.5 & 5.0 & 135.2 & 47.4 & 54.7 \\
\hline 53023.5216 & 0.559 & 249.2 & 10.4 & 23.7 & 419.2 & 8.9 & -27.8 \\
\hline 53027.5315 & 0.344 & 441.2 & 14.8 & -26.2 & 25.6 & 8.6 & 3.5 \\
\hline 53028.5271 & 0.786 & 118.3 & 17.8 & 13.2 & 674.8 & 17.5 & 12.3 \\
\hline 53028.7270 & 0.875 & 165.0 & 8.0 & 6.9 & 591.8 & 37.4 & 21.7 \\
\hline 53029.5269 & 0.231 & 518.6 & 9.8 & 20.2 & 19.6 & 32.4 & 54.4 \\
\hline 53030.5260 & 0.676 & 131.5 & 10.3 & 11.4 & 631.7 & 15.5 & -1.9 \\
\hline 53030.7169 & 0.761 & 96.7 & 10.5 & -3.6 & 683.5 & 20.2 & 13.2 \\
\hline 53031.5200 & 0.118 & 475.7 & 22.6 & 40.1 & 118.6 & 20.8 & 44.2 \\
\hline 53032.5246 & 0.565 & 234.0 & 18.9 & 15.5 & 487.5 & 48.1 & 28.3 \\
\hline 53032.7225 & 0.653 & 146.3 & 3.9 & 12.1 & 569.6 & 21.2 & -38.7 \\
\hline 53033.5391 & 0.017 & 346.1 & 6.3 & 25.4 & 209.5 & 20.0 & -69.8 \\
\hline $53710.7543^{\mathrm{a}}$ & 0.347 & 539.9 & 39.3 & 75.4 & 31.7 & 33.4 & 5.4 \\
\hline
\end{tabular}


Table 1 - Continued

\begin{tabular}{|c|c|c|c|c|c|c|c|}
\hline $\begin{array}{c}\text { Date } \\
\text { (HJD-2,400,000) }\end{array}$ & $\begin{array}{l}\text { Orbital } \\
\text { Phase }\end{array}$ & $\begin{array}{c}V_{1} \\
\left(\mathrm{~km} \mathrm{~s}^{-1}\right)\end{array}$ & $\begin{array}{c}\sigma_{1} \\
\left(\mathrm{~km} \mathrm{~s}^{-1}\right)\end{array}$ & $\begin{array}{l}(O-C)_{1} \\
\left(\mathrm{~km} \mathrm{~s}^{-1}\right)\end{array}$ & $\begin{array}{c}V_{2} \\
\left(\mathrm{~km} \mathrm{~s}^{-1}\right)\end{array}$ & $\begin{array}{c}\sigma_{2} \\
\left(\mathrm{~km} \mathrm{~s}^{-1}\right)\end{array}$ & $\begin{array}{l}(O-C)_{2} \\
\left(\mathrm{~km} \mathrm{~s}^{-1}\right)\end{array}$ \\
\hline $53711.7167^{\mathrm{a}}$ & 0.776 & 77.1 & 52.7 & -29.5 & 688.2 & 79.4 & $23 . .6$ \\
\hline 53748.5493 & 0.165 & 458.6 & 11.7 & -13.4 & 14.7 & 21.9 & 4.2 \\
\hline 53752.6857 & 0.006 & 304.4 & 25.3 & -2.3 & 282.6 & 31.5 & -22.0 \\
\hline 53753.5655 & 0.397 & 454.1 & 15.9 & 32.0 & 73.8 & 23.6 & -28.8 \\
\hline 53755.7542 & 0.371 & 438.2 & 9.6 & -8.5 & -3.4 & 20.9 & -62.5 \\
\hline $53765.6205^{\mathrm{a}}$ & 0.761 & 55.1 & 21.2 & -49.6 & 623.4 & 64.7 & -44.4 \\
\hline $53766.6131^{\mathrm{a}}$ & 0.203 & 526.3 & 43.6 & 40.6 & -54.9 & 78.2 & -37.6 \\
\hline
\end{tabular}

${ }^{\text {a }}$ Data from the echelle spectrograph on the $2.5 \mathrm{~m}$ DuPont telescope at Las Campanas.

Table 2. Circular Orbital Solutions for [L72] LH 54-425

\begin{tabular}{|c|c|c|}
\hline Element & Combined Solution & Spectroscopic Solution \\
\hline$P$ (days) & $2.24741 \pm 0.00004$ & $2.24746 \pm 0.00010$ \\
\hline$T_{\mathrm{IC}, 1}(\mathrm{HJD}-2,400,000)$. & $53029.007 \pm 0.003$ & $53029.016 \pm 0.007$ \\
\hline$K_{1}\left(\mathrm{~km} \mathrm{~s}^{-1}\right) \ldots \ldots \ldots$ & $201.6 \pm 3.8$ & $210.8 \pm 3.0$ \\
\hline$K_{2}\left(\mathrm{~km} \mathrm{~s}^{-1}\right) \ldots$ & $359.1 \pm 4.5$ & $350.8 \pm 5.2$ \\
\hline$\gamma_{1}\left(\mathrm{~km} \mathrm{~s}^{-1}\right) \ldots$ & $299.7 \pm 0.6$ & $303.1 \pm 2.0$ \\
\hline$\gamma_{2}\left(\mathrm{~km} \mathrm{~s}^{-1}\right) \ldots$ & $316.7 \pm 2.2$ & $317.7 \pm 3.3$ \\
\hline rms (Primary) $\left(\mathrm{km} \mathrm{s}^{-1}\right)$ & 19.4 & 21.9 \\
\hline rms (Secondary) $\left(\mathrm{km} \mathrm{s}^{-1}\right)$ & 33.2 & 32.8 \\
\hline rms (Photometry) (mag). & 0.007 & $\cdots$ \\
\hline
\end{tabular}

Table 3. Tomographic Spectral Reconstruction Parameters for [L72] LH 54-425

\begin{tabular}{lcc}
\hline \hline \multicolumn{1}{c}{ Parameter } & Primary & Secondary \\
\hline Spectral Type & O3 V & O5 V \\
$T_{\text {eff }}(\mathrm{kK})$ & $45 \pm 1$ & $41 \pm 1$ \\
$\log g(\mathrm{cgs})$ & $4.0 \pm 0.2$ & $4.0 \pm 0.2$ \\
$V \sin i\left(\mathrm{~km} \mathrm{~s}^{-1}\right)$ & $197 \pm 5$ & $182 \pm 8$ \\
$F_{2} / F_{1}(\mathrm{blue})$ & \multicolumn{2}{c}{$0.45 \pm 0.05$} \\
\hline
\end{tabular}


Table 4. ELC Model Parameters for [L72] LH 54-425

\begin{tabular}{|c|c|c|c|c|}
\hline \multirow{2}{*}{$\begin{array}{c}\text { Parameter } \\
\ldots\end{array}$} & \multicolumn{2}{|c|}{ Distorted Secondary } & \multicolumn{2}{|c|}{ Distorted Primary } \\
\hline & Primary & Secondary & Primary & Secondary \\
\hline Inclination (deg) & \multicolumn{2}{|c|}{$52+2$} & \multicolumn{2}{|c|}{$55 \pm 1$} \\
\hline$M\left(M_{\odot}\right)$ & $53_{-4}^{+7}$ & $32_{-2}^{+4}$ & $47 \pm 2$ & $28 \pm 1$ \\
\hline$R_{\text {eff }}\left(R_{\odot}\right)$ & $11.0_{-0.3}^{+0.7}$ & $9.7_{-0.2}^{+1.0}$ & $11.4 \pm 0.1$ & $8.1 \pm 0.1$ \\
\hline$R_{\text {pole }}^{\mathrm{a}}\left(R_{\odot}\right)$ & $10.7_{-0.2}^{+0.3}$ & $9.2_{-0.2}^{+0.3}$ & $10.9 \pm 0.1$ & $7.8 \pm 0.1$ \\
\hline$R_{\text {point }} \mathrm{b}\left(R_{\odot}\right)$ & $11.7_{-0.2}^{+0.4}$ & $11.1_{-0.2}^{+0.2}$ & $12.3 \pm 0.2$ & $8.6 \pm 0.1$ \\
\hline$V_{\mathrm{sync}} \sin i\left(\mathrm{~km} \mathrm{~s}^{-1}\right)$ & $197 \pm 5$ & $173 \pm 7$ & $209 \pm 5$ & $148 \pm 6$ \\
\hline $\log g(\cos )$ & $4.08_{-0.01}^{+0.01}$ & $3.94_{-0.01}^{+0.02}$ & $4.00 \pm 0.02$ & $4.07 \pm 0.01$ \\
\hline Filling Factor & $0.66_{-0.02}^{+0.04}$ & $0.80_{-0.02}^{+0.08}$ & $0.72_{-0.02}^{+0.03}$ & $0.64_{-0.02}^{+0.03}$ \\
\hline$a_{\text {tot }}\left(R_{\odot}\right)$ & \multicolumn{2}{|c|}{$31.6_{-0.6}^{+1.0}$} & \multicolumn{2}{|c|}{$30.4 \pm 0.4$} \\
\hline$F_{2} / F_{1}$ (blue) & \multicolumn{2}{|c|}{$0.70_{-0.01}^{+0.05}$} & \multicolumn{2}{|c|}{$0.46 \pm 0.02$} \\
\hline
\end{tabular}

${ }^{\text {aPolar radius. }}$

${ }^{\mathrm{b}}$ Radius toward the inner Lagrangian point. 


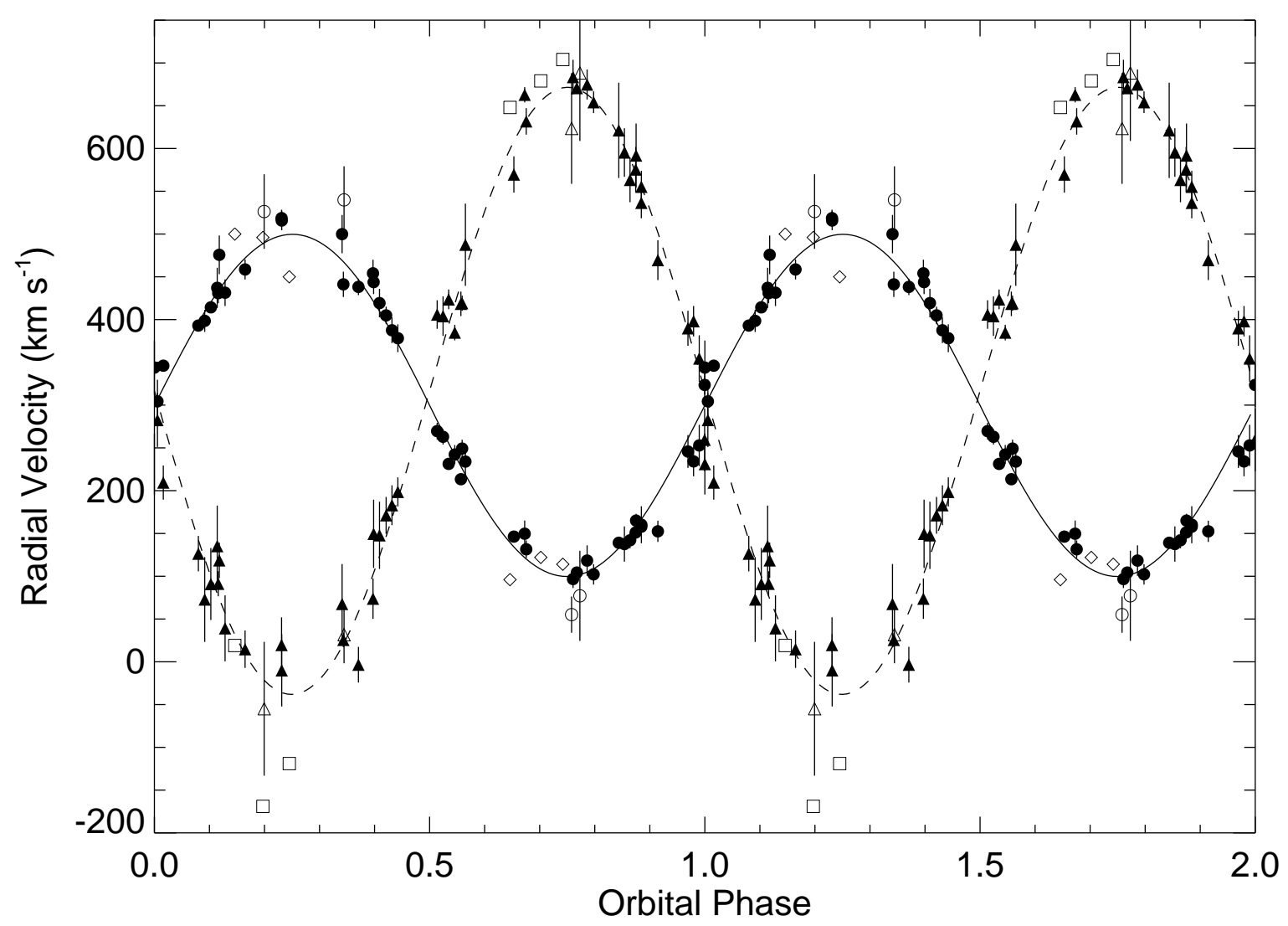

Fig. 1.- Radial velocity curves for [L72] LH 54-425. Primary radial velocities are shown by filled dots and secondary radial velocities are represented by filled triangles with associated uncertainties shown as line segments for both. The open circles and triangles represent the echelle data from Las Campanas. The open diamonds and squares are the radial velocity measurements from Ostrov (2002) for the primary and secondary, respectively. The Ostrov data were not used in model fits. The solid line is the best combined fit solution for the primary, and the dotted line is the same for the secondary. 


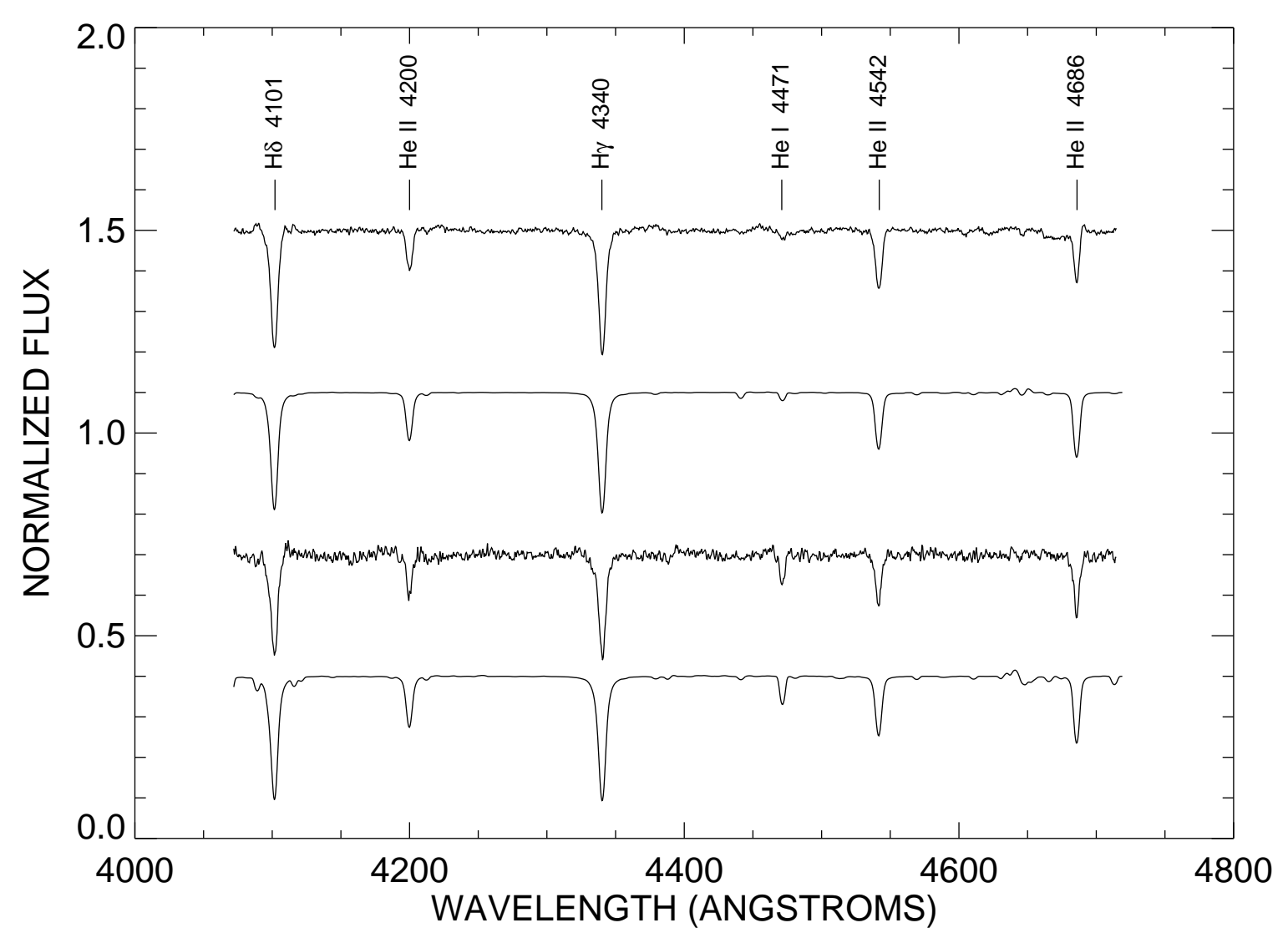

Fig. 2.- Tomographic reconstructions of the components of [L72] LH 54-425 based on 48 spectra obtained from 2003 through 2006 at CTIO. Plotted from top to bottom are line identifications with vertical tick marks, the primary spectrum, the model primary spectrum (Lanz \& Hubeny 2003), the secondary spectrum, and the model secondary spectrum. The stellar parameters for the model spectra are given in Table 3 . 


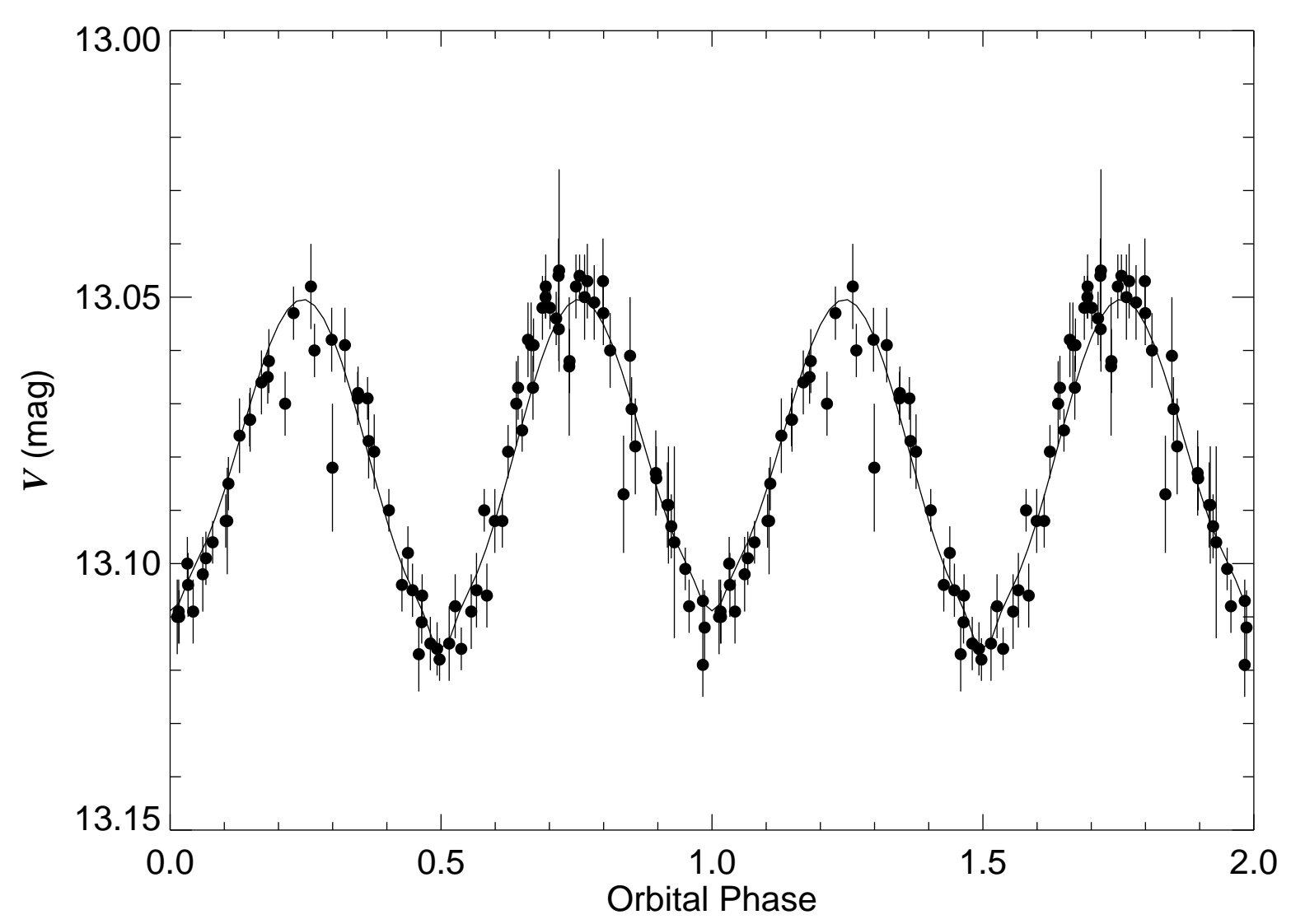

Fig. 3.- $V$-band light curve for [L72] LH 54-425. These data were taken from Ostrov (2002) and are presented here in phase according to our best combined solution. The model is the solid line and the data are represented by filled dots with $V$ uncertainties shown by line segments. Phase zero corresponds to inferior conjunction of the primary star (which differs by 0.5 phase from that adopted by Ostrov 2002). 


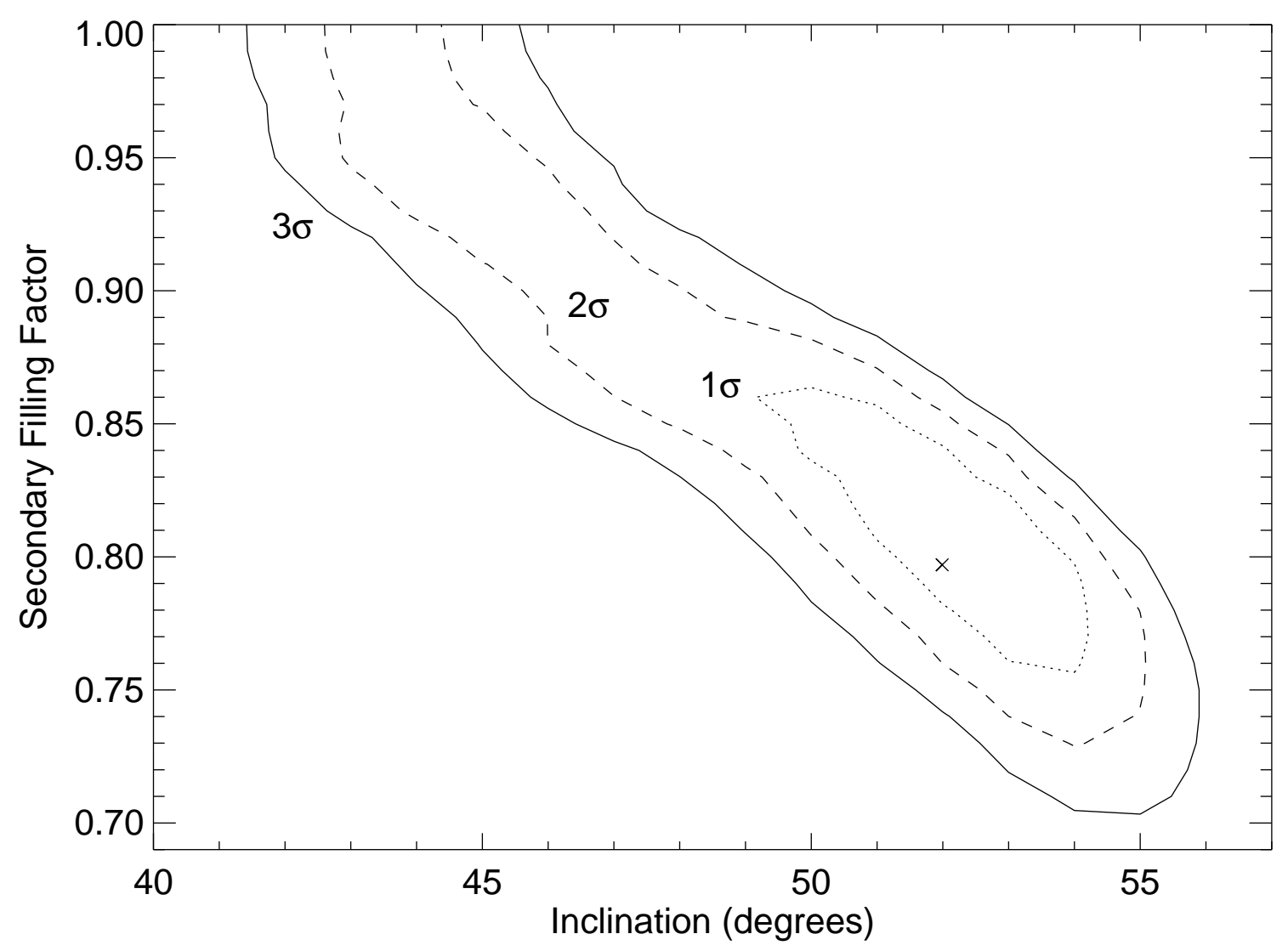

Fig. 4. - $\chi^{2}$ surface contours of the residuals from the combined velocity and light curve data as a function of orbital inclination and secondary Roche-lobe filling factor of the best fit solution. The best fit position is represented by the " $\mathrm{x}$ " inside the $1-\sigma$ contour. The range of filling factor is quite large, while the inclination is reasonably well constrained. 


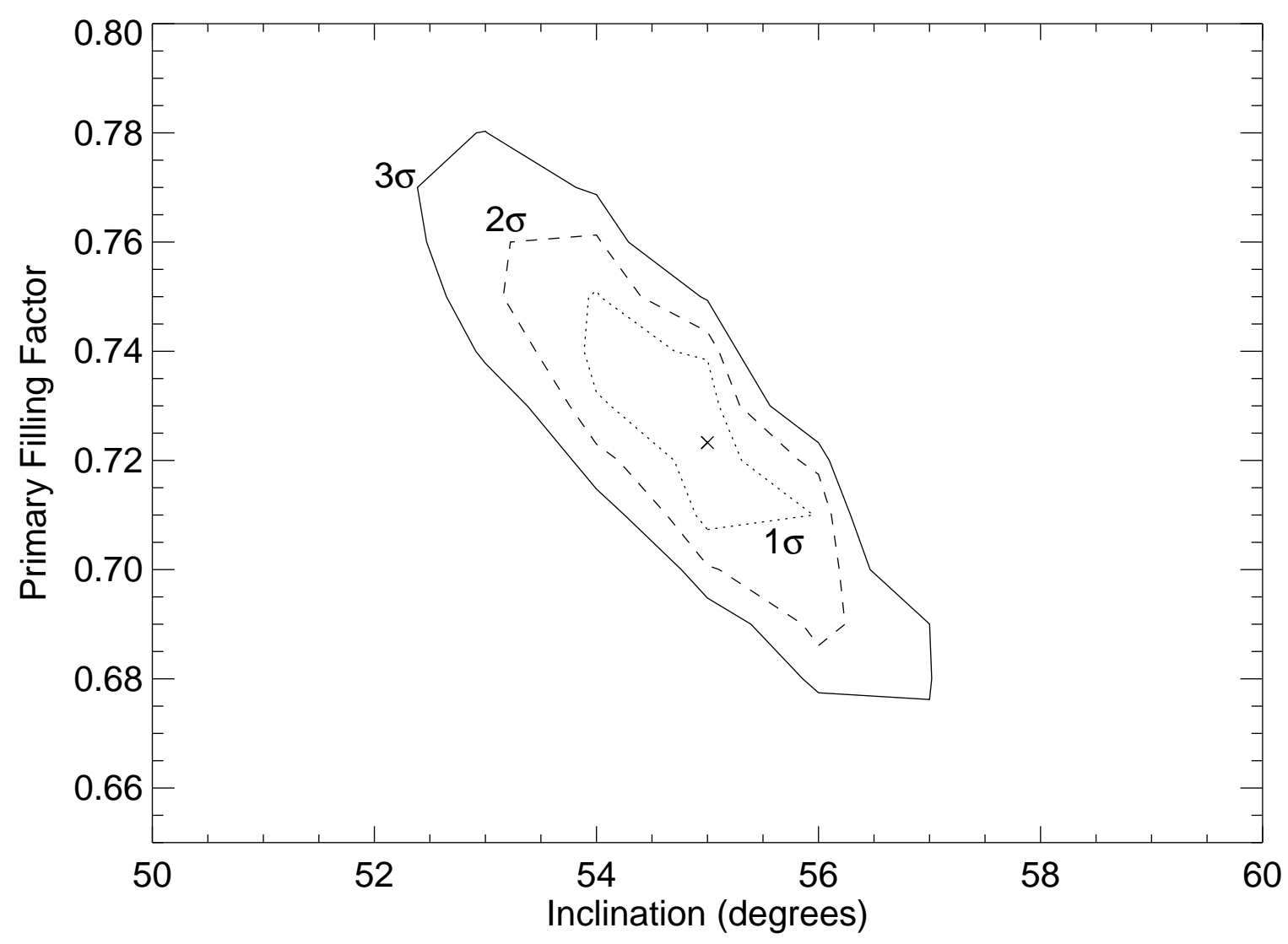

Fig. 5.- $\chi^{2}$ surface contours of the combined fit residuals as a function of the orbital inclination and Roche-lobe filling factor of the primary star for the case where the primary is the more tidally distorted star. The best fit position is represented by the " $\mathrm{x}$ " inside the $1-\sigma$ contour. The parameter space is more confined than for the best fit, and the contours are smaller around the best inclination for this configuration. 


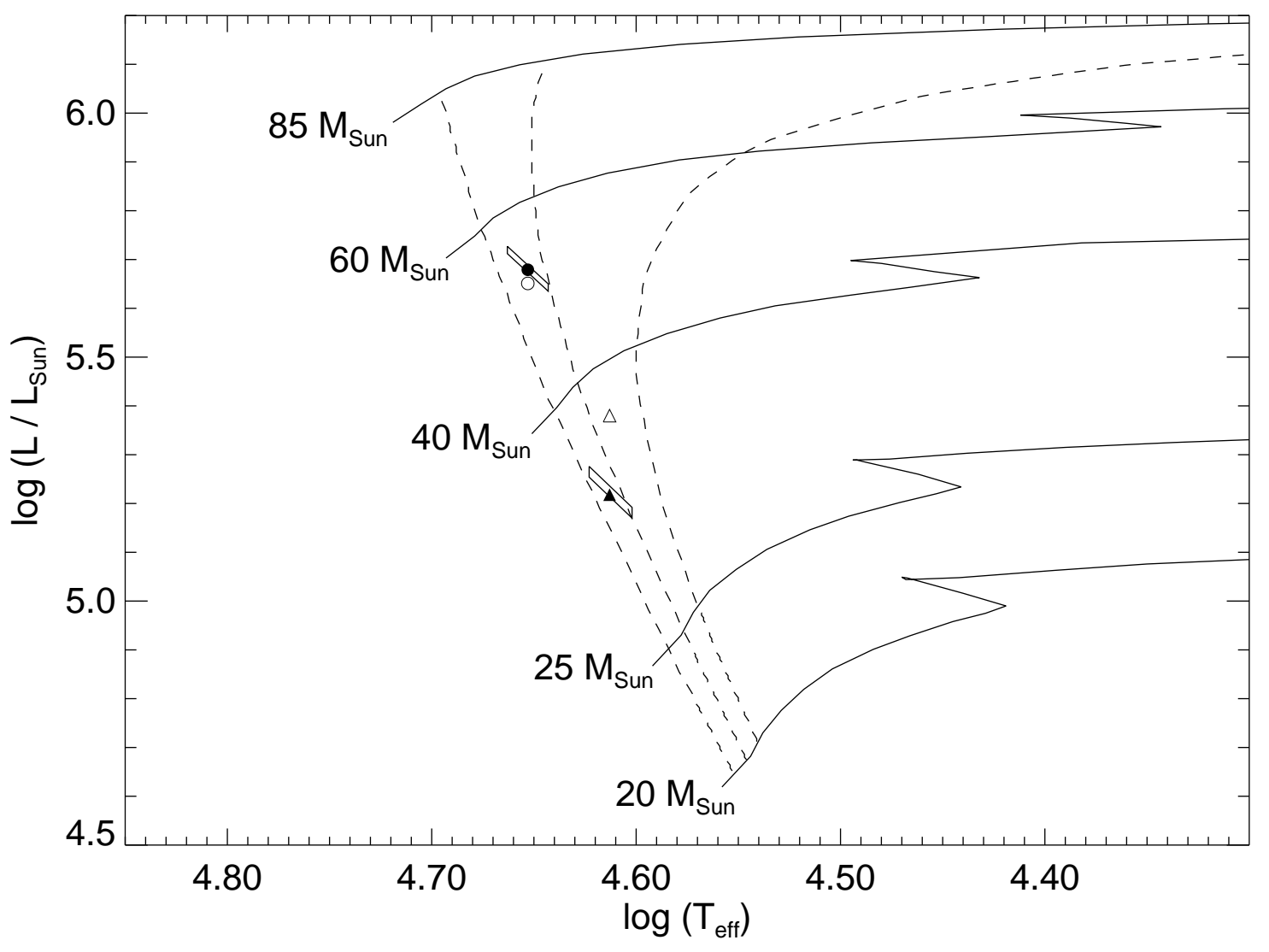

Fig. 6.- An H-R diagram showing the location of the primary star (filled circle) and secondary star (filled triangle) of [L72] LH 54-425 for our fit where the primary is the more tidally distorted star. The open symbols represent the fit where the secondary is the more tidally distorted star. Also plotted are evolutionary tracks for stars of various masses from Schaerer et al. (1993) for an LMC metallicity. The boxes around the filled data points correspond to the 1- $\sigma$ uncertainties in the derived values of $T_{\text {eff }}$ and $R$ from the fits where the primary star is the more tidally distorted star. The vertical dashed lines correspond to isochrones from Lejeune \& Schaerer (2001), also for an LMC metallicity, of 1, $\sim 2$ and $\sim 3.2$ Myr going from left to right. The positions of the two stars are consistent with an age of $\sim 1.5$ Myr. 